medRxiv preprint doi: https://doi.org/10.1101/2020.12.18.20248459; this version posted December 20, 2020. The copyright holder for this preprint (which was not certified by peer review) is the author/funder, who has granted medRxiv a license to display the preprint in perpetuity.

It is made available under a CC-BY-NC 4.0 International license .

\title{
1 Common X-chromosome variants are associated with Parkinson's disease risk
}

\section{Running title: X-chromosome and Parkinson's disease risk}

4 Authors: Yann Le Guen ${ }^{1, *}, \mathrm{PhD}$, Valerio Napolioni ${ }^{2, *}, \mathrm{PhD}$, Michael E. Belloy ${ }^{1}, \mathrm{PhD}$, Eric Yu ${ }^{3,4}$,

5 BSc, Lynne Krohn ${ }^{3,4}$, MSc, Jennifer A. Ruskey ${ }^{4,5}$, MSc, Ziv Gan-Or ${ }^{3,4,5}$, MD, PhD, Gabriel

6 Kennedy $^{1}$, BSc, Sarah J. Eger ${ }^{1}$, BA, and Michael D. Greicius ${ }^{1}$, MD, MPH.

$8 \quad{ }^{1}$ Department of Neurology and Neurological Sciences, Stanford University, Stanford, CA, 94305, USA

$9{ }^{2}$ School of Biosciences and Veterinary Medicine, University of Camerino, Camerino, 62032, Italy

$10 \quad{ }^{3}$ Department of Human Genetics, McGill University, Montréal, QC, Canada

$11{ }^{4}$ Montreal Neurological Institute and Hospital, McGill University, Montréal, QC, Canada

12 Department of Neurology and Neurosurgery, McGill University, Montréal, QC, Canada

13 *Y.L.G and V.N. contributed equally to this work.

14

\section{Corresponding Author}

16 Yann Le Guen

17 Department of Neurology and Neurological Sciences - Greicius lab

18 Stanford University

19 290 Jane Stanford Way, E265, CA 94305-5090

20 Tel: 6506662696

21 Email: yleguen@stanford.edu 
medRxiv preprint doi: https://doi.org/10.1101/2020.12.18.20248459; this version posted December 20, 2020. The copyright holder for this preprint (which was not certified by peer review) is the author/funder, who has granted medRxiv a license to display the preprint in perpetuity.

It is made available under a CC-BY-NC 4.0 International license .

\section{$1 \quad$ Abstract (limit 250 words)}

2 Objective: Identify genetic variants on the X-chromosome associated with Parkinson's disease 3 (PD) risk.

4 Methods: We performed an X-chromosome-wide association study (XWAS) of PD risk by meta-

5 analyzing results from sex-stratified analyses. To avoid spurious associations, we designed a

6 specific harmonization pipeline for the X-chromosome and focused on a European ancestry

7 sample. We included 11,324 cases, 280,060 controls, and 5,379 proxy cases, based on parental

8 history of PD. Additionally, we tested the association of significant variants with: (i) PD risk in an

9 independent replication with 1,564 cases and 2,467 controls, and (ii) putamen volume in 33,360

10 individuals from the UK Biobank.

11 Results: In the discovery meta-analysis, we identified: rs7066890 ( $\mathrm{OR}=1.10$ [1.06-1.14];

$\left.12 \mathrm{P}=2.2 \times 10^{-9}\right)$ intron of $G P M 6 B$, and $\mathrm{rs} 28602900\left(\mathrm{OR}=1.10[1.07-1.14] ; \mathrm{P}=1.6 \times 10^{-8}\right)$ in a high gene

13 density region including RPL10, ATP6A1, FAM50A, PLXNA3. The rs28602900 association with

$14 \mathrm{PD}$ was replicated $(\mathrm{OR}=1.16[1.03-1.30] ; \mathrm{P}=0.016)$ and shown to colocalize with a significant

15 expression quantitative locus (eQTL) regulating RPL10 expression in the putamen and other brain

16 tissues in GTEx. Additionally, the rs28602900 locus was found to be associated with reduced brain

17 putamen volume. No results reached genome-wide significance in the sex-stratified analyses.

18 Interpretation: We report the first XWAS of PD and identify two genome-wide significant loci.

19 The rs28602900 association replicated in an independent PD dataset and showed concordant

20 effects in its association with putamen volume. Critically, rs26802900 is a significant eQTL of

$21 R P L 10$. These results support a role for ribosomal proteins in PD pathogenesis and show that the

22 X-chromosome contributes to PD genetic risk. 


\section{Introduction}

2 Parkinson's Disease (PD), a neurodegenerative disorder characterized by a broad range of motor

3 and non-motor symptoms, is thought to be caused by a combination of aging, genetics and

4 environmental factors. PD affects the nervous system at multiple levels from the enteric nervous

5 system to the cortex, but prominent motor symptoms associated with PD are linked to the

6 substantia nigra and striatum. PD heritability has been estimated at 34\% in a twin study ${ }^{1}$ and 16

7 to $36 \%$ in genome-wide association studies (GWAS) ${ }^{2}$. The latter estimates were derived from

8 autosomal variants and did not include the genetic contribution of sex chromosomes. Similarly,

9 the latest PD GWAS meta-analysis, which identified 90 common genetic variants associated with

10 PD risk, focused solely on autosomes ${ }^{2}$. The effect of common sex chromosome variants on PD

11 risk remains unexplored, despite the relative risk for PD being 1.5 times greater in males than in

12 females ${ }^{3-6}$. Various mechanisms have been proposed to account for this sex difference in PD risk,

13 including sex hormone levels and other environmental and genetic factors influenced by biological

14 sex $^{7,8}$. To date, a few studies have identified variants or loci on the X-chromosome linked to PD,

15 notably, a linkage analysis in PD multiplex families which identified the PARK12 locus ${ }^{9}$. However

16 this locus is quite large and has not yet been linked to a gene ${ }^{10}$. Whole-exome sequencing studies

17 in pedigree and single case reports have linked loss of function mutations on $R A B 39 B$ with

18 Waisman syndrome, an X-linked early-onset, $\alpha$-synucleinopathy with Parkinsonism and

19 intellectual disability ${ }^{11-14}$.

20 Though the $\mathrm{X}$-chromosome is $155 \mathrm{Mb}$ and accounts for $5 \%$ of the human genome, it remains

21 excluded from the vast majority of GWAS because it requires different quality control than

22 autosomal chromosomes ${ }^{15}$ due to the way it is inherited. Females inherit one X-chromosome from

23 each parent, while males only inherit a maternal copy and outside of pseudoautomosal regions,

24 male $\mathrm{X}$-chromosomes do not undergo any recombination during meiosis. This causes the $\mathrm{X}$ -

25 chromosome to have reduced genetic diversity. Notably, the X-chromosome structure is more

26 sensitive to historical events such as population bottleneck, sex-biased demographics events, and

27 has a different mutation rates than autosomes ${ }^{16}$. Additionally, until recently, genotyping arrays

28 were poorly designed for the X-chromosome ${ }^{15}$.

29 Our work addresses this gap by conducting the first PD X-chromosome-wide association study

30 (XWAS). Specifically, we performed meta-analyses including all publicly available PD cohorts 
1 with X-chromosome data, as well as the UK Biobank cohort, in which we used both diagnosed PD

2 individuals and proxy-cases based on parental history of PD. The use of this PD proxy phenotype 3 was recently shown to provide increased power for the discovery of PD genetic risk factors ${ }^{2}$. We

4 first meta-analyzed males and females separately to identify potential sex specific effects. Then,

5 to increase statistical power, we meta-analyzed across the sexes to identify variants influencing

6 PD risk in a sex-independent fashion. Variants that reached genome-wide significance were tested

7 for replication in an independent PD meta-analysis and tested for association with putamen volume

8 in the UK Biobank, and functionally annotated in gene expression datasets through colocalization

9 analyses, leading to the identification of a putatively causal gene for PD that may provide novel

10 targets for future therapeutic development.

\section{Methods}

\section{Samples}

14 For the discovery PD meta-analysis, we used two types of samples: PD cohorts published in

15 previous studies and the UK Biobank, using both directly diagnosed PD and a proxy phenotype 16 based on parental history of PD. The PD cohorts include the International PD Genomics 17 Consortium (IPDGC) NeuroX dataset ${ }^{17}$, the National Institute of Neurological Disorders and 18 Stroke (NINDS) PD dataset ${ }^{18}$, the Autopsy-Confirmed PD GWAS Consortium (APDGC) dataset 19 genotyped by the Center for Inherited Disease Research (CIDR), the NeuroGenetics Research 20 Consortium (NGRC) dataset ${ }^{19}$, the UK PD Consortium and The Wellcome Trust Case Control 21 Consortium $2^{20}$, referred to here as EBI MERGE. The whole-genome sequencing (WGS) data 22 were obtained from the Accelerating Medicines Partnership - Parkinson's Disease (AMP-PD), 23 composed of the following cohorts: New Discovery of Biomarkers (BioFIND), the Harvard 24 Biomarker Study (HBS), the Parkinson's Progression Markers Initiative (PPMI) ${ }^{21}$, and the 25 Parkinson's Disease Biomarkers Program (PDBP). Table 1 shows a detailed description of the 26 cohorts including genotyping platform, number of X-chromosome single nucleotide 27 polymorphisms (SNPs) and demographics.

28 In the replication, we meta-analyzed two PD datasets (Table 2): the first composed of the 29 Parkinson's Disease Cognitive Genetics Consortium (PDCGC) ${ }^{22}$ and healthy individuals from the 30 Adult Change in Thought (ACT) longitudinal cohort ${ }^{23}$, referred to here as PDCGC \& ACT, and 
1 the second from McGill University (McGill) including samples from the Quebec Parkinson

2 Network $(\mathrm{QPN})^{24}$.

3 The putamen volume analysis consisted of individuals with magnetic resonance imaging (MRI)

4 scans that were successfully processed by the UK Biobank FSL pipeline ${ }^{25}$ as of January 2020.

5 The putamen volume used in our analysis corresponds to the average of the left and right

6 hemispheres. UK Biobank details regarding the MRI scan parameters, and image quality control

7 are described elsewhere ${ }^{26,27}$.

8 The current study protocol was granted an exemption by the Stanford University institutional

9 review board because the analyses were carried out on deidentified, off-the-shelf data; therefore,

10 further informed consent was not required.

\section{Quality control, ancestry determination and imputation}

13 To perform the first PD XWAS, we introduced a quality control and harmonization pipeline of X14 chromosome SNP-array data from homogenous ancestry samples prior to $\mathrm{X}$ imputation. 15 Autosomal variants were extracted from the SNP array genetic data and processed through our 16 harmonization pipeline in several stages. First, we removed multi-allelic SNPs, SNPs located on 17 structural variants and duplicated or monomorphic SNPs. The list of multi-allelic SNPs or SNPs 18 located on common structural variants was created using Tri-Typer 28 and gnomAD ${ }^{29}$. 19 Additionally, we removed variants located on potential probe polymorphisms identified from 20 gnomAD. These are defined as SNPs for which the probe may have variable affinity due to the 21 presence of other SNP(s) within $20 \mathrm{bp}$ and with minor allele frequency (MAF) above 1\%. SNPs 22 were checked for consistency with the Haplotype reference consortium (HRC) panel ${ }^{30}$ using a 23 checking script developed by the McCarthy group (well.ox.ac.uk/ wrayner/tools/). This script 24 enabled us to flip SNPs reported on the incorrect strand and excluded palindromic SNPs and SNPs 25 with more than $10 \%$ MAF difference from the reference panel.

26 Using these quality control SNPs as inputs, we further excluded SNPs with a genotyping rate $<$ $2795 \%$ and Hardy-Weinberg equilibrium (HWE) $\mathrm{p}<10^{-5}$ in controls and also removed individuals 28 with more than 5\% genotype missingness. Next, the individual ancestry was determined with 29 SNPweights v.2.1 ${ }^{31}$ using reference populations from the 1000 Genomes Consortium ${ }^{32}$. By 30 applying an ancestry percentage cut-off $>75 \%$, the samples were stratified into the five super 31 populations, South-Asians, East-Asians, Americans, Africans and Europeans. Since most of the 
1 samples belonged to the European population, we also determined their percent ancestry to three 2 major ethnicities, Northwestern, Southeastern, and Ashkenazi Jewish, using reference populations 3 available from SNPweights v.2.1. European subjects were stratified into the above-mentioned 4 ethnicities when their ancestry percentage was greater than $50 \%$. Further analyses were focused 5 on the Northwestern European (NWE) subsample, which represents the vast majority of subjects and provided a highly homogenous sample. Two main reasons explain the more extreme population structure on the X-chromosome compared to autosomes: (i) the X-chromosome has a 8 smaller effective population size and thus the rate of genetic drift of X-linked loci is amplified, (ii) local adaptation will lead to a higher levels of differentiation between geographically isolated population ${ }^{33}$. As such, to better control our population structure, we decided to be stringent and 11 restrict our analyses to NWE subjects.

12 Additionally, we merged all genotype quality control autosomes and performed an identity-by13 descent on the whole NWE sample to identify duplicates and first-degree relatives. If the diagnosis 14 was incongruent between cohorts for a duplicate subject, we chose the cohort with a PD diagnosis. 15 Otherwise, we kept the subject's data in the cohort with the following order of priority based on 16 sequencing coverage of the X chromosome: AMP-AD WGS, APDGC, EBI MERGE, NINDS, 17 NGRC, and IPDGC. Similarly, for first degree relatives, given the direction of the sex bias in PD 18 risk, we prioritized the inclusion of a male relative over a female one. Additionally, we kept cases 19 over controls, used the same order of priority for cohort selection when relatives had the same 20 diagnosis and chose the older of the two relatives if they were controls in the same cohort.

21 The $\mathrm{X}$ chromosome variants underwent a similar harmonization pipeline as the autosomes. We 22 excluded multi-allelic SNPs, SNPs within structural variations, and potential probe polymorphism 23 SNPs. Additionally, our analysis excluded the pseudo autosomal regions of the X chromosome 24 and used only the NWE subsample as derived above. Several steps were performed to avoid 25 spurious findings: (1) variants with less than 95\% genotyping rate and (2) individuals with more 26 than 5\% genotype missingness were excluded. (3) Reported sex was checked using PLINK1.9 34 27 --check-sex flag, with 0.4 max value for females and 0.94 min value for men and all individuals 28 with a discordant sex label were excluded. (4) Heterozygous SNPs in males were set as missing in 29 males, while (5) SNPs with differential missingness between PD cases and controls were removed $30\left(\mathrm{p}<10^{-5}\right)$. (6) HWE was tested in female controls and SNPs with $\mathrm{p}<10^{-5}$ were removed. (7) Any 31 monomorphic SNPs that remained were removed. (8) Differential missingness and differential 
1 MAF between males and females were both tested and SNPs with $\mathrm{p}<10^{-5}$, for either one of the

2 tests, were excluded. Finally, as for the autosomes, (9) remaining SNPs were checked for

3 consistency with the HRC panel, flipping palindromic SNPs and excluding SNPs with more than

$4 \quad 10 \%$ MAF difference from the reference panel.

5 The EBI MERGE dataset, which includes two SNP arrays, had these quality control steps

6 performed separately on each array and the remaining SNPs were merged and re-processed

7 through the whole harmonization pipeline. Similarly, PDCGC \& ACT autosomes were first

8 harmonized separately, then merged and re-harmonized together before ancestry determination

9 and PCA computation. By contrast, the X-chromosome data were imputed separately and merged

10 after imputation, due to a low X coverage of the NeuroX array used in PDCGC that resulted in

11 only 111 overlapping ACT-PDCGC variants after harmonization.

12 The remaining $\mathrm{X}$ chromosome quality controlled SNPs were imputed on the Michigan Imputation

13 Server (v1.2.4) ${ }^{35}$, which uses minimac 4 for imputation and improved X chromosome support.

14 The following parameters were selected: reference panel HRC r1.1 2016 (hg19) with the EUR

15 subpanel, phasing Eagle v2.4, r-square imputation score cut off 0.3. Before the XWAS with PD

16 status, SNPs with MAF $<0.01$ or genotyping rate $<95 \%$ were removed in WGS and imputed data.

17 Genetic principal components (PC) were computed within the NWE subsample for each cohort to

18 account for population stratification in the downstream analysis ${ }^{36}$. PCs were inspected for each

19 cohort and indicated a homogenous sample.

20 The UK Biobank genotyping, imputation and quality control data are described in detail elsewhere

2137 . We considered the 33,360 British individuals with MRI scan, whose genetic data successfully

22 passed the UK Biobank quality control and were not diagnosed with PD, nor had a parent with

23 PD.

\section{Study-level analyses and meta-analysis}

26 To identify sex-specific effects, we first performed sex-stratified meta-analyses separating males

27 and females. We then performed a combined meta-analysis across all sex-stratified cohorts, to

28 identify sex-independent effects with increased statistical power.

29 The male meta-analysis included 5,745 cases and 4,875 controls of NWE ancestry from PD

30 cohorts, 706 PD cases and 128,275 controls from the UK Biobank unrelated British males, and

313,551 proxy male cases and 143,316 controls using the father's PD status in unrelated British 
1 females. The rationale is that the female dosage, in this case, corresponds to the probability of the

2 father carrying the variant and testing it against his PD status. Put differently, if a female has two 3 copies of the effect allele, then her father is a carrier; if a female has one copy then there is $50 \%$ 4 chance that her father is a carrier, if a female has no copies then her father is a non-carrier. The 5 female meta-analysis included 3,104 cases and 4,925 controls of NWE ancestry from PD cohorts, 396 PD cases and 146,867 controls from the UK Biobank unrelated British females, and 1,828 proxy female cases and 126,447 controls using the mother's PD status in unrelated British males.

8 In this scenario, the mother must carry at least one copy for a male carrier and zero or no more than one copy for a male non-carrier. We excluded directly diagnosed PD subjects from the proxy

10 phenotype analysis.

11 The associations were estimated with PLINKv2.0 ${ }^{34}$ using the $-g l m$ flag, which performs a 12 standard logistic regression for case/control phenotype. For each cohort, we covaried by the age 13 provided in each study and the 10 PCs computed within the NWE sample. In each separate study, 14 cases and controls were roughly age matched, with cases slightly older than controls (Table 1).

15 This small age difference per cohort resulted in a small positive effect of age on PD risk that was 16 adjusted for, except in the EBI MERGE cohort where age was unavailable. The age provided 17 varied across studies between age at onset, age at clinical diagnosis or age at death for cases, and 18 age at last exam or age at death for controls. To address this heterogeneity, we ran the analysis 19 with and without age as a covariate and the loci passing the suggestive threshold did not change.

20 For the UK Biobank, the covariates used were the first 10 PCs provided by the UK Biobank and 21 the age at last visit of the participant. Finally, we meta-analyzed all XWAS summary statistics 22 using fixed effect meta-analysis as implemented in GWAMA ${ }^{38}$. Additionally, GWAMA tests for 23 sex-heterogenous effects by combining male and female effects into a chi-squared distribution 24 with one degree of freedom (assuming the same allelic effect in males and females) and testing the 25 null hypothesis that the association is the same in males and females. The genome-wide 26 significance threshold was set at $\mathrm{p}<5 \times 10^{-8}$ and the suggestive significance threshold at $\mathrm{p}<10^{-5}$, 27 corresponding to X-chromosome-wide correction.

28 We queried the expression quantitative loci (eQTL) among our XWAS loci in brain tissues of the 29 Genotype-Tissue Expression (GTEx) v8 ${ }^{39}$. GTEx (gtexportal.org) includes bulk RNASeq data 30 from 12 brain tissues types: amydgala $(n=129)$, anterior cingulate cortex $(n=147)$, caudate $(n=$ 31 194), cerebellar hemisphere $(n=175)$, cerebellum $(n=209)$, cortex $(n=205)$, frontal cortex $(n=$ 
1 175), hippocampus $(\mathrm{n}=165)$, hypothalamus $(\mathrm{n}=170)$, nucleus accumbens $(\mathrm{n}=202)$, putamen $(\mathrm{n}$

$2=170)$, and substantia nigra $(n=114)$. Additionally, we queried the Braineac database 40

3 (braineac.org), which includes $n=134$ individuals with up to 12 different brain regions sequenced

4 with RNA exon arrays. The eQTLs nominal significance, as well as false discovery rate (FDR)

5 correction ${ }^{41}$, were performed by GTEx ${ }^{39}$ and Braineac ${ }^{40}$. Colocalization was performed using the

6 R package $\operatorname{coloc}^{42}$ and we report the posterior probability of colocalization (PP4) between PD and

7 QTL associations in Figures S1-S4. Colocalization between XWA and eQTL results were

8 inspected with locuscompare ${ }^{43}$ for all FDR significant results from GTEx.

9 We tested the association of the significant loci with putamen volume using a Bayesian linear

10 mixed model as implemented in LMM-BOLT ${ }^{44}$. In this second validation analysis males and

11 females were analyzed together coding males as $0 / 2$ and females as $0 / 1 / 2$. We adjusted the analysis

12 by brain volume, age at MRI scan, genotyping array, sex, and 10 PCs accounting for population

13 stratification.

\section{Results}

\section{Parkinson's Disease X-chromosome discovery meta-analyses}

17 We meta-analyzed all XWAS summary results in males, females, and in both sexes combined (Fig

18 1). In visually inspecting each cohort's QQ plots and genomic control lambdas, we observed no 19 inflation $(\lambda=0.983-1.042)$. As a sensitivity analysis, we also conducted the analysis without

20 accounting for any covariates. The top associations remained unchanged and lambdas remained

21 within the normal range $(\lambda=0.971-1.069)$.

22 In the combined sex meta-analysis $(\lambda=1.041)$, two loci reached genome-wide significance with

23 top SNPs: rs7066890 near GPM6B $\left(\mathrm{OR}=1.10, \mathrm{MAF}=0.16, \mathrm{p}=2.2 \times 10^{-9}\right)$, rs 28602900 near

$24 R P L 10\left(\mathrm{OR}=1.10, \mathrm{MAF}=0.12, \mathrm{p}=1.6 \times 10^{-8}\right)$. For each SNP, the minor allele was associated with

25 increased risk and in all analyses described below the rsID will refer to the minor allele. These two

26 SNPs had a concordant direction of effect across cohorts (Fig 2). The first SNP is an intron of

$27 G P M 6 B$, and the second SNP is located in a region with a high gene density (39 genes, within +/$28400 \mathrm{~kb})$.

29 For the sex-stratified analyses $\left(\lambda_{\text {male }}=1.004, \lambda_{\text {female }}=0.980\right)$ there were no genome-wide significant

30 results. Loci passing the suggestive, X-chromosome-wide significance threshold are shown in 
medRxiv preprint doi: https://doi.org/10.1101/2020.12.18.20248459; this version posted December 20, 2020. The copyright holder for this preprint (which was not certified by peer review) is the author/funder, who has granted medRxiv a license to display the preprint in perpetuity.

It is made available under a CC-BY-NC 4.0 International license .

1 Figure 1 and detailed in Table 3. The two genome-wide significant loci from the combined sex

2 meta-analysis had a non-differentiated effect across sex.

\section{Independent PD replication meta-analysis}

5 In two independent datasets, we tested the association with PD of the two lead SNPs from the 6 genome-wide significant loci. The rs28602900 association with PD significantly replicated $(\mathrm{OR}=$

7 1.16, $\mathrm{p}=0.016)$, whereas rs7066890 was imputed in only one of the two datasets and showed a

8 discordant direction of effect $(\mathrm{OR}=0.96, \mathrm{p}=0.54)$. Thus, the rs7066890 association did not

9 replicate but remained genome-wide significant after meta-analysis with the replication $(\mathrm{p}=$

$101.1 \times 10^{-8}$, Table 3).

\section{Functional annotation via eQTL analysis}

12 The identified loci lie in regions with multiple genes, so we sought to identify the potentially causal 13 genes through eQTL analysis. To this aim, we queried the genome-wide significant SNPs in the

14 GTEx v8 ${ }^{39}$ and Braineac ${ }^{40}$ databases, which both contain gene expression data from brain tissues 15 and include eQTL analyses of X-chromosome SNPs.

16 All the following associations for rs28602900 were FDR significant in GTEx. rs28602900 was

17 associated with decreased RPL10 expression in 11 queried brain tissues. rs28602900 was also 18 associated with decreased PLXNA3 expression in 6 brain tissues (cerebellum, frontal cortex, 19 cortex, putamen, cerebellar hemisphere, anterior cingulate cortex); increased FAM50A expression 20 in 4 brain tissues (cerebellum, hypothalamus, cerebellar hemisphere, nucleus accumbens); 21 increased ATP6AP1 expression in the frontal cortex only; and decreased DNASE1L1 expression 22 in the cerebellum only. In Braineac, rs28602900 was significantly associated with decreased 23 RPL10 and PLXNA3 average expression across brain tissues ( $\mathrm{p}<0.05$, FDR-corrected). The 24 rs28602900 association with RPL10 was also FDR significant at the single tissue level in the 25 cerebellum and temporal cortex tissues. No FDR-significant eQTL associations were observed in 26 brain tissues for rs7066890 (GPM6B locus) in GTEx or Braineac.

27 The RPL10 eQTL colocalized across 11 brain tissues with the locus associated with PD risk (PP4 28 between 0.90 and 0.97, Fig S1). The colocalization is illustrated for the putamen (Fig 3A). The 
1 FAM50A eQTLs in 4 brain tissues and the ATP6AP1 eQTL in frontal cortex all also colocalized

2 with the identified PD risk locus (PP4 between 0.90 and 0.95, and lower PP4 in other brain tissues,

3 Fig S1). On the other hand, the PLXNA3 eQTL association is driven by a nearby stronger PLXNA3

4 eQTL, which is in low linkage disequilibrium with the locus associated with PD risk. This is

5 illustrated for the putamen (PP4 $=0$, Fig 3B) and occurred similarly in the 5 other brain tissues

6 with a significant eQTL for PLXNA3 (PP4 $\approx 0)$. Similarly, the DNASE1L1 eQTL in the cerebellum

7 was driven by a locus in low linkage disequilibrium with our PD risk association locus (PP4 = $8 \quad 0.4)$.

\section{Association with putamen volume in the UK Biobank}

11 To further assess and validate the role of the two genome-wide significant loci, we tested their

12 association in UK Biobank with the putamen volume, an established PD biomarker ${ }^{45}$. In a sample

13 composed of 32,896 British individuals, excluding PD cases and PD-proxy-cases, the lead SNP in

14 the RPL10 locus, rs28602900, was significantly associated with decreased putamen volume $(\beta=$ -

$1511.01, \sigma=4.35, \mathrm{p}=0.011$ ). The lead SNP in the GPM6B locus, rs7066890, was not significantly

16 associated with putamen volume.

\section{Discussion}

19 Our study demonstrates a genome-wide significant association of PD with two X-chromosome

20 loci and paves the way for further XWAS studies to decipher the role of common X-chromosome

21 variants in neurodegenerative diseases. This analysis expands our understanding of the genes

22 involved in PD. These new loci will help refine polygenic risk scores to determine an individual's

23 genetic susceptibility to PD. We have also leveraged eQTL data to focus attention on a gene new

24 to PD which may have important pathogenic implications.

25 The RPL10 locus has the strongest supports across our analyses and was replicated in an 26 independent PD meta-analysis. rs28602900, the top SNP in the locus, was significantly associated

27 with increased risk in our combined sex meta-analysis and with reduced putamen volume in the 28 UKB analysis. While this locus is in a high gene-density region, we were able to rank-order the 29 list of potentially causal genes through functional annotations based on gene expression analysis.

30 The most significant brain eQTLs from this locus point towards a regulation of RPL10, but 
1 FAM50A, ATP6AP1 could also be putative candidates based on this evidence. The expression 2 association signals were stronger for RPL10 than for FAM50A or ATP6AP1 and for RPL10 reached 3 FDR-corrected significance across all 11 brain tissues in GTEx. In addition, the expression 4 analyses in Braineac also showed a significant association between rs28602900 and the average 5 RPL10 expression across all brain tissues (but no significant association for FAM50A or ATP6AP1). In both GTEx and Braineac, RPL10 expression was lower in carriers of the rs28602900 minor allele. RPL10, ribosomal protein L10, has been associated with intellectual disability ${ }^{46}$, cerebellar hypoplasia ${ }^{47}$, microcephaly ${ }^{48}$, and autism spectrum disorder ${ }^{49,50}$. More generally, in a gene and protein network analysis of PD, Alzheimer's disease and amyotrophic lateral sclerosis,

10 Monti et al. emphasized that 6 out of 25 PD-specific proteins were ribosomal ${ }^{51}$. Additionally, 11 ribosomal proteins interact with $L R R K 2$ and elevate kinase activity ${ }^{52}$. Specifically, the ribosomal 12 protein, RPS15, was shown to be a key pathogenic LRRK2 substrate mediating dopamine neuron 13 degeneration ${ }^{52}$. The reduced expression of several ribosomal proteins in PD brain tissues is 14 associated with alpha-synuclein aggregation ${ }^{53}$.

15 The second locus, near GPM6B, did not replicate in an independent PD meta-analysis and did not 16 have any eQTL signals in brain tissue that survived FDR correction. The top SNP at the locus is 17 intronic in GPM6B, whose associated protein is highly expressed in brain. The locus is also 18 situated near a number of other brain-expressed proteins such as OFD1, TRAPPC2, and GEMIN8, 19 but for the time being, the gene associated with PD risk at this locus remains uncertain.

20 Interestingly, a linkage analysis in multiplex PD families identified a large PD X-linked region, 21 PARK12, spanning Xq21-q25 9 . Our analysis highlights a well-defined signal within this region, 22 with a lead variant missense of $R T L 9\left(\mathrm{OR}=1.15, \mathrm{p}=9.0 \times 10^{-8}\right.$, Table 3). Linkage studies are well23 powered to discover genetic loci associated with disease and reduce the risk of false positives due 24 to population structure, but this approach often cannot fine-map the causal gene. Our 25 complementary XWAS approach allowed us to refine this original signal and emphasized the most 26 likely polymorphism increasing disease risk at this locus.

27 The main limitation of our study is that while we have three suggestive loci and two loci meeting 28 the genome-wide significance threshold - including one replicating in an independent PD meta29 analysis and strongly supported by eQTL and imaging analyses - we do not have access to 30 additional replication datasets to validate more of these loci. Two large datasets used in the recent 31 PD GWAS ${ }^{2}$ could not, unfortunately, be used here because access to the data is restricted by the 
1 investigative teams. When additional datasets become available, future studies will allow further

2 validation and extension of the findings presented here. In addition, the inclusion of UK Biobank

3 proxy phenotypes that rely on participant reports of family history likely injects some diagnostic

4 uncertainty into those data, but we believe this is outweighed by the gain in statistical power from

5 being able to include this large cohort in our analyses.

6 In conclusion, our work provides compelling evidence for two novel loci on the $\mathrm{X}$ chromosome

7 that increase risk for PD and that can be used to refine polygenic risk scores of PD. Most critically,

8 these results focus attention on a new-to-PD gene, RPL10, that should further increase enthusiasm

9 for the role of ribosomal proteins in PD pathogenesis ${ }^{51,52}$. 
medRxiv preprint doi: https://doi.org/10.1101/2020.12.18.20248459; this version posted December 20, 2020. The copyright holder for this preprint (which was not certified by peer review) is the author/funder, who has granted medRxiv a license to display the preprint in perpetuity.

It is made available under a CC-BY-NC 4.0 International license .

\section{Acknowledgments}

Funding for this study was provided by the Iqbal Farrukh \& Asad Jamal Center for Cognitive Health in Aging, and the NIH (AG060747 and AG047366). ZGO is supported by the Fonds de recherche du Québec - Santé (FRQS) Chercheurs-boursiers award, in collaboration with Parkinson Quebec, and by the Young Investigator Award by Parkinson Canada.

We would like to thank all of the subjects who donated their time and biological samples to be a part of this study.

\section{Acknowledgment for the use of PD datasets}

PD data included the International Parkinson's Disease Consortium (IPDGC), NeuroX dataset provided through dbGaP accession phs000918.v1.p1. We thank the IPDGC and the funding source 100064005/11348001. National Institute of Neurological Disorders and Stroke, National Institute on Aging, National Institutes of Health, Bethesda, MD, and United States Army Medical Research Acquisition Activity (USAMRAA), Fort Detrick, MD/Department of Defense, DC, USA. (PI Andrew Singleton, PhD, and Mike Nalls, PhD). PD data also included the National Institute of Neurological Disorders and Stroke (NINDS) Parkinson's Disease dataset provided through dbGaP accession phs001172.v1.p2 (PI Andrew Singleton, PhD, and John Hardy, PhD). PD data also included the Autopsy-Confirmed Parkinson Disease GWAS Consortium (APDGC) dataset provided through dbGaP accession (phs000394.v1.p1) (PI Jeffery Vance, MD, PhD, Collaborators: Thomas Beach, MD, PhD, Karen Marder, MD, MPH, Tatiana M. Foroud, Ted M. Dawson, MD, PhD, Matthew P. Frosch, MD, PhD, Dennis W. Dickson, MD, Samuel M. Goldman, MD, MPH, Harry Vinters, MD, Deborah Mash, PhD, James Leverenz, MD, Thomas J. Montine, $\mathrm{MD}, \mathrm{PhD}$, John Q. Trojanowski, MD, PhD, Vivianna Van Deerlin, MD, PhD) with funding sources: P50 NS071674-01S1 NIH, X01 HG006076-01 NIH, P50 NS072187-01S2 NIH, and genotyping center Johns Hopkins University Center for Inherited Disease Research (CIDR), Baltimore, MD, USA (HHSN268201100011I NHGRI and NIH). PD data also included the Genome-Wide Association Study of Parkinson Disease: Genes and Environment dataset provided through dbGaP accession (phs000196.v3.p1) (PI Haydeh Payami, PhD, and co-investigators: John Nutt, MD, Cyrus Zabetian, MS, MD, Stewart Factor, DO, Eric Molho, MD, Donald Higgins, MD) with funding source: 5R01NS36960-10 and genotyping center Johns Hopkins University Center for Inherited Disease Research (CIDR), Baltimore, MD, USA (HHSN268200782096C NIH). PD data also included the Parkinson's Disease Cognitive Genetics Consortium (PDCGC) Stage I, 
medRxiv preprint doi: https://doi.org/10.1101/2020.12.18.20248459; this version posted December 20, 2020. The copyright holder for this

1 NeuroX Dataset (phs001664.v1.p1) for which we acknowledge the Pacific Northwest Udall Center 2 (P50 NS062684). Healthy individuals from the Adult Change in Thought (ACT) study, which is a 3 longitudinal prospective cohort study that began in 1994 (UO1 AG006781, UO1 HG004610, UO1 4 HG006375, U01 HG008657). PD data also included the dataset generated by the Wellcome Trust 5 Case-Control Consortium 2 (WTCCC2) from UK patients with Parkinson's disease and UK control individuals from the 1958 Birth Cohort and National Blood Service (available through the EBI, accession EGAD00000000022 and EGAD00000000057). This study makes use of data generated by the Wellcome Trust Case-Control Consortium. A full list of the investigators who contributed to the generation of the data is available from www.wtccc.org.uk. Funding for the project was provided by the Wellcome Trust under award 076113, 085475 and 090355. Part of the data used in the preparation of this article were obtained from the AMP PD Knowledge Platform. For up-to-date information on the study, https:/www.amp-pd.org.AMP PD - a public-

13 private partnership - is managed by the FNIH and funded by Celgene, GSK, the Michael J. Fox

14 Foundation for Parkinson's Research, the National Institute of Neurological Disorders and Stroke, 15 Pfizer, Sanofi, and Verily. The AMP-AD data included clinical data and biosamples obtained from 16 the Fox Investigation for New Discovery of Biomarkers (BioFIND), the Harvard Biomarker Study 17 (HBS), the Parkinson's Progression Markers Initiative (PPMI), and the Parkinson's Disease 18 Biomarkers Program (PDBP). BioFIND is sponsored by The Michael J. Fox Foundation for 19 Parkinson's Research (MJFF) with support from the National Institute for Neurological Disorders 20 and Stroke (NINDS). The BioFIND Investigators have not participated in reviewing the data 21 analysis or content of the manuscript. For up-to-date information on the study, visit 22 michaeljfox.org/biofin. Harvard NeuroDiscovery Biomarker Study (HBS) is a collaboration of 23 HBS investigators funded through philanthropy and NIH and Non-NIH funding sources. A full list 24 of HBS investigator can found at https://neurodiscovery.harvard.edu/biomarkers-core. The HBS 25 Investigators have not participated in reviewing the data analysis or content of the manuscript. 26 Parkinson's Progression Markers Initiative (PPMI) - a public-private partnership - is funded by 27 the Michael J. Fox Foundation for Parkinson's Research and funding partners, including Abbvie, 28 Allergan, Amathus therapeutics, Avid radiopharmaceuticals, Biogen, BioLegend, Bristol-Myers 29 Squibb, Celgene, Denali, GE Healthcare, Genentech (Roche group), GSK, Janssen Neuroscience 30 (Johnson-Johnson group), Lilly, Lundbeck, Merck, MSD, Pfizer, Piramal, Prevail, Roche, Sanofi 31 Genzyme, Servier, Takeda, Teva, UCB, Verily, Voyager Therapeutics, Golub Capital. The PPMI 
medRxiv preprint doi: https://doi.org/10.1101/2020.12.18.20248459; this version posted December 20, 2020. The copyright holder for this preprint (which was not certified by peer review) is the author/funder, who has granted medRxiv a license to display the preprint in perpetuity.

It is made available under a CC-BY-NC 4.0 International license .

1 Investigators have not participated in reviewing the data analysis or content of the manuscript. For

2 up-to-date information on the study, visit www.ppmi-info.org. Parkinson's Disease Biomarker

3 Program (PDBP) consortium is supported by the NINDS at the National Institutes of Health. A

4 full list of PDBP investigators can be found at https://pdbp.ninds.nih.gov/policy. The PDBP

5 Investigators have not participated in reviewing the data analysis or content of the manuscript. The

6 McGill cohort was financially supported by grants from the Michael J. Fox Foundation, the

7 Canadian Consortium on Neurodegeneration in Aging (CCNA), the Canada First Research

8 Excellence Fund (CFREF), awarded to McGill University for the Healthy Brains for Healthy Lives

9 initiative (HBHL), and Parkinson Canada. The access to part of the participants for this research

10 has been made possible thanks to the Quebec Parkinson's Network (http://rpq-qpn.ca/en/).

\section{Acknowledgement for the use of the UK Biobank data}

13 This research has been conducted using the UK Biobank Resource under Application Number 1445420.

\section{Author contributions}

17 Y.L.G processed and analyzed data, designed analyses, designed study, and wrote paper. V.N. 18 processed data, designed study, and supervised work. M.E.B., S.J.E., and G.K. processed data.

19 E.Y., L.K., J.A.R., and Z.G.O acquired and processed data. M.D.G designed study, supervised 20 work, and wrote paper. Y.L.G and V.N. contributed equally to this work. 


\section{References}

2

1. Wirdefeldt K, Gatz M, Reynolds CA, et al. Heritability of Parkinson disease in Swedish twins: a longitudinal study. Neurobiology of Aging 2011;32(10):1923.e1-1923.e8.

2. Nalls MA, Blauwendraat C, Vallerga CL, et al. Identification of novel risk loci, causal insights, and heritable risk for Parkinson's disease: a meta-analysis of genome-wide association studies. The Lancet Neurology 2019;18(12):1091-1102.

3. Van Den Eeden SK, Tanner CM, Bernstein AL, et al. Incidence of Parkinson's disease: variation by age, gender, and race/ethnicity. American journal of epidemiology 2003;157(11):1015-22.

4. Wooten GF, Currie LJ, Bovbjerg VE, et al. Are men at greater risk for Parkinson's disease than women? Journal of Neurology, Neurosurgery and Psychiatry 2004;75(4):637-639.

5. Taylor KSM, Cook JA, Counsell CE. Heterogeneity in male to female risk for Parkinson's disease. Journal of Neurology, Neurosurgery and Psychiatry 2007;78(8):905-906.

6. Miller IN, Cronin-Golomb A. Gender differences in Parkinson's disease: Clinical characteristics and cognition. Movement Disorders 2010;25(16):2695-2703.

7. Gillies GE, Pienaar IS, Vohra S, Qamhawi Z. Sex differences in Parkinson's disease. Frontiers in Neuroendocrinology 2014;35(3):370-384.

8. Jurado-Coronel JC, Cabezas R, Ávila Rodríguez MF, et al. Sex differences in Parkinson's disease: Features on clinical symptoms, treatment outcome, sexual hormones and genetics. Frontiers in Neuroendocrinology 2018;50(June 2017):18-30.

9. Pankratz N, Nichols WC, Uniacke SK, et al. Genome-wide linkage analysis and evidence of geneby-gene interactions in a sample of 362 multiplex Parkinson disease families. Human Molecular Genetics 2003;12(20):2599-2608.

10. Klein C, Westenberger A. Genetics of Parkinson's Disease. Cold Spring Harb Perspect Med 2012;2(1):a008888.

11. Wilson GR, Sim JCH, McLean C, et al. Mutations in RAB39B cause X-linked intellectual disability and early-onset parkinson disease with $\alpha$-synuclein pathology. American Journal of Human Genetics 2014;95(6):729-735.

12. Lesage S, Bras J, Cormier-Dequaire F, et al. Loss-of-function mutations in RAB39B are associated with typical early-onset Parkinson disease [Internet]. Neurology Genetics 2015;1(1)[cited 2020 Dec 10 ] Available from: https://ng.neurology.org/content/1/1/e9

13. Mata IF, Jang Y, Kim C-H, et al. The RAB39B p.G192R mutation causes X-linked dominant Parkinson's disease. Molecular Neurodegeneration 2015;10(1):50.

14. Ciammola A, Carrera P, Fonzo AD, et al. X-linked Parkinsonism with Intellectual Disability caused by novel mutations and somatic mosaicism in RAB39B gene. Parkinsonism \& Related Disorders 2017;44:142-146. 
15. Wise AL, Gyi L, Manolio TA. eXclusion: Toward Integrating the X Chromosome in Genome-wide Association Analyses. The American Journal of Human Genetics 2013;92(5):643-647.

16. Gottipati S, Arbiza L, Siepel A, et al. Analyses of X-linked and autosomal genetic variation in population-scale whole genome sequencing. Nature Genetics 2011;43(8):741-743.

17. Nalls MA, Pankratz N, Lill CM, et al. Large-scale meta-analysis of genome-wide association data identifies six new risk loci for Parkinson's disease. Nature Genetics 2014;46(9):989-993.

18. Fung HC, Scholz S, Matarin M, et al. Genome-wide genotyping in Parkinson's disease and neurologically normal controls: first stage analysis and public release of data. Lancet Neurology 2006;5(11):911-916.

19. McCulloch CC, Kay DM, Factor SA, et al. Exploring gene-environment interactions in Parkinson's disease. Human Genetics 2008;123(3):257-265.

20. Spencer CCA, Plagnol V, Strange A, et al. Dissection of the genetics of Parkinson's disease identifies an additional association 5' of SNCA and multiple associated haplotypes at 17q21. Human Molecular Genetics 2011;20(2):345-353.

21. Nalls MA, Keller MF, Hernandez DG, et al. Baseline genetic associations in the Parkinson's Progression Markers Initiative (PPMI). Movement Disorders 2016;31(1):79-85.

22. Mata IF, Johnson CO, Leverenz JB, et al. Large-scale exploratory genetic analysis of cognitive impairment in Parkinson's disease. Neurobiology of Aging 2017;56:211.e1-211.e7.

23. Montine TJ, Sonnen JA, Montine KS, et al. Adult Changes in Thought study: dementia is an individually varying convergent syndrome with prevalent clinically silent diseases that may be modified by some commonly used therapeutics. Curr Alzheimer Res 2012;9(6):718-723.

24. Gan-Or Z, Rao T, Leveille E, et al. The Quebec Parkinson Network: A Researcher-Patient Matching Platform and Multimodal Biorepository. Journal of Parkinson's Disease 2020;10(1):301-313.

25. Alfaro-Almagro F, Jenkinson M, Bangerter NK, et al. Image processing and Quality Control for the first 10,000 brain imaging datasets from UK Biobank. NeuroImage 2018;166:400-424.

26. Miller KL, Alfaro-Almagro F, Bangerter NK, et al. Multimodal population brain imaging in the UK Biobank prospective epidemiological study. Nature neuroscience 2016;19(11):1523-1536.

27. Le Guen Y, Leroy F, Philippe C, et al. Enhancer Locus in ch14q23.1 Modulates Brain Asymmetric Temporal Regions Involved in Language Processing. Cerebral Cortex 2020;1-11.

28. Franke L, de Kovel CGF, Aulchenko YS, et al. Detection, Imputation, and Association Analysis of Small Deletions and Null Alleles on Oligonucleotide Arrays. American Journal of Human Genetics 2008;82(6):1316-1333.

29. Karczewski KJ, Francioli LC, Tiao G, et al. Variation across 141,456 human exomes and genomes reveals the spectrum of loss-of-function intolerance across human protein-coding genes. bioRxiv $2019 ; 531210$. 
30. McCarthy S, Das S, Kretzschmar W, et al. A reference panel of 64,976 haplotypes for genotype imputation. Nature Genetics 2016;48(10):1279-1283.

3 31. Chen CY, Pollack S, Hunter DJ, et al. Improved ancestry inference using weights from external reference panels. Bioinformatics 2013;29(11):1399-1406.

32. Auton A, Abecasis GR, Altshuler DM, et al. A global reference for human genetic variation. Nature 2015;526(7571):68-74.

33. Lambert CA, Connelly CF, Madeoy J, et al. Highly Punctuated Patterns of Population Structure on the X Chromosome and Implications for African Evolutionary History. The American Journal of Human Genetics 2010;86(1):34-44.

34. Chang CC, Chow CC, Tellier LC, et al. Second-generation PLINK: rising to the challenge of larger and richer datasets. GigaScience 2015;4(1):7.

12 35. Das S, Forer L, Schönherr S, et al. Next-generation genotype imputation service and methods.

13 Nature Genetics 2016;48(10):1284-1287.

14 36. Price AL, Patterson NJ, Plenge RM, et al. Principal components analysis corrects for stratification in genome-wide association studies. Nature Genetics 2006;38(8):904-909.

37. Bycroft C, Freeman C, Petkova D, et al. The UK Biobank resource with deep phenotyping and genomic data. Nature 2018;562(7726):203-209.

38. Mägi R, Morris AP. GWAMA: software for genome-wide association meta-analysis. BMC Bioinformatics 2010;11(1):288.

39. GTEx Consortium. Genetic effects on gene expression across human tissues. Nature 2017;550(7675):204-213.

40. Ramasamy A, Trabzuni D, Guelfi S, et al. Genetic variability in the regulation of gene expression in ten regions of the human brain. Nature Neuroscience 2014;17(10):1418-1428.

41. Storey JD, Tibshirani R. Statistical significance for genomewide studies. PNAS 2003;100(16):9440-9445.

42. Giambartolomei C, Vukcevic D, Schadt EE, et al. Bayesian Test for Colocalisation between Pairs of Genetic Association Studies Using Summary Statistics. PLOS Genetics 2014;10(5):e1004383.

43. Liu B, Gloudemans MJ, Rao AS, et al. Abundant associations with gene expression complicate GWAS follow-up. Nat Genet 2019;51(5):768-769.

44. Loh P-R, Tucker G, Bulik-Sullivan BK, et al. Efficient Bayesian mixed-model analysis increases association power in large cohorts. Nature Genetics 2015;47(3):284-290.

45. Pitcher TL, Melzer TR, MacAskill MR, et al. Reduced striatal volumes in Parkinson's disease: a magnetic resonance imaging study. Translational Neurodegeneration 2012;1(1):17. syndromic Intellectual Disability. American Journal of Medical Genetics, Part A 2015;167(8):19081912. 
medRxiv preprint doi: https://doi.org/10.1101/2020.12.18.20248459; this version posted December 20, 2020. The copyright holder for this preprint (which was not certified by peer review) is the author/funder, who has granted medRxiv a license to display the preprint in perpetuity.

It is made available under a CC-BY-NC 4.0 International license .

1

2

3

4

5
47. Zanni G, Kalscheuer VM, Friedrich A, et al. A Novel Mutation in RPL10 (Ribosomal Protein L10) Causes X-Linked Intellectual Disability, Cerebellar Hypoplasia, and Spondylo-Epiphyseal Dysplasia. Human Mutation 2015;36(12):1155-1158.

48. Brooks SS, Wall AL, Golzio C, et al. A novel ribosomopathy caused by dysfunction of RPL10 disrupts neurodevelopment and causes X-linked microcephaly in humans. Genetics 2014;198(2):723-733.

49. Chiocchetti A, Pakalapati G, Duketis E, et al. Mutation and expression analyses of the ribosomal protein gene RPL10 in an extended German sample of patients with autism spectrum disorder. American Journal of Medical Genetics, Part A 2011;155(6):1472-1475.

50. Gong X, Delorme R, Fauchereau F, et al. An investigation of ribosomal protein L10 gene in autism spectrum disorders. BMC Medical Genetics 2009;10:1-5.

51. Monti C, Colugnat I, Lopiano L, et al. Network analysis identifies disease-specific pathways for parkinson's disease. Molecular Neurobiology 2018;55(1):370-381.

52. Martin I, Kim JW, Lee BD, et al. Ribosomal protein s15 phosphorylation mediates LRRK2 neurodegeneration in parkinson's disease. Cell 2014;157(2):472-485.

53. Garcia-Esparcia P, Hernández-Ortega K, Koneti A, et al. Altered machinery of protein synthesis is region- and stage-dependent and is associated with $\alpha$-synuclein oligomers in Parkinson's disease. Acta Neuropathologica Communications 2015;3(1):1-25. 
medRxiv preprint doi: https://doi.org/10.1101/2020.12.18.20248459; this version posted December 20, 2020. The copyright holder for this preprint (which was not certified by peer review) is the author/funder, who has granted medRxiv a license to display the preprint in perpetuity.

It is made available under a CC-BY-NC 4.0 International license .
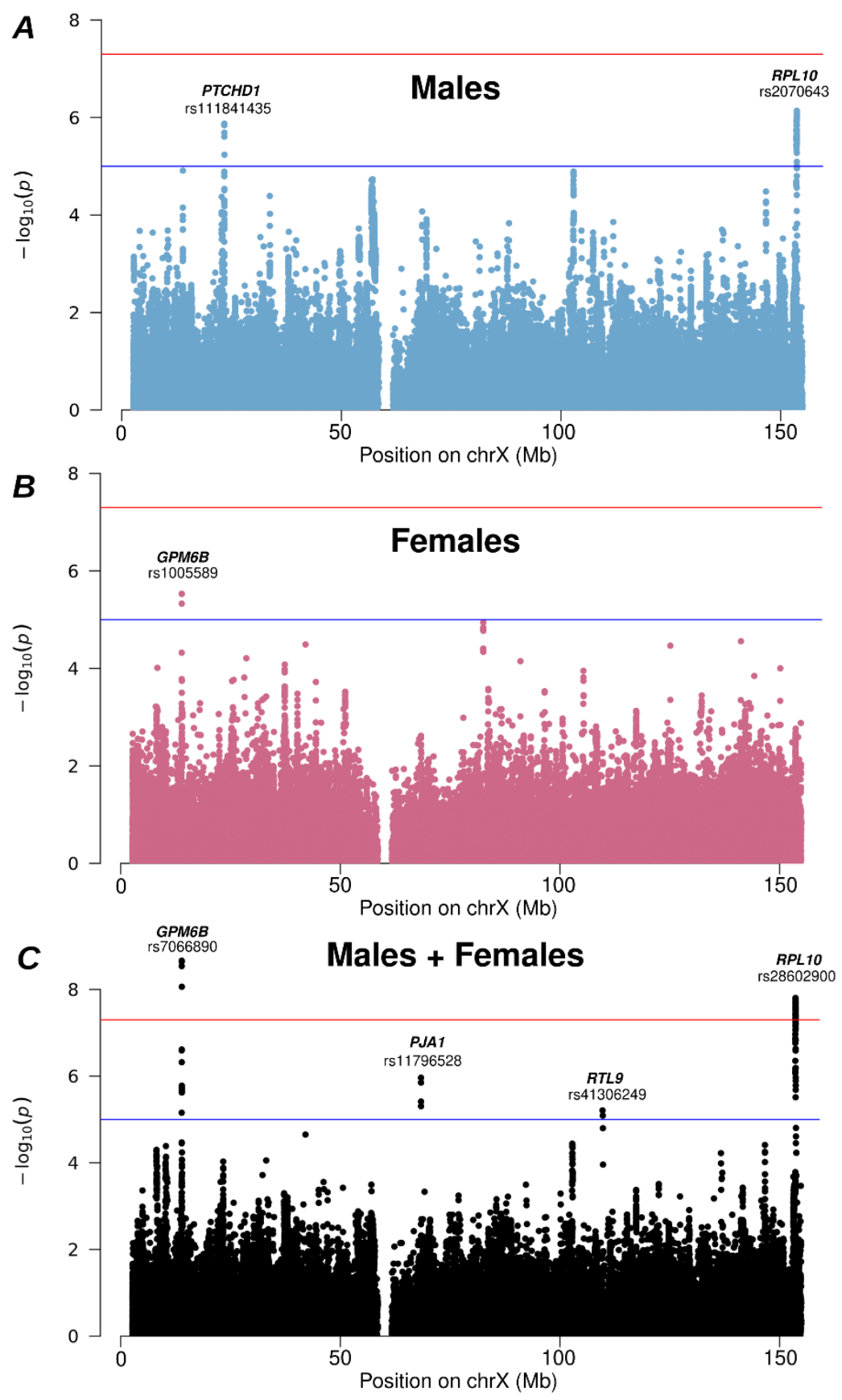

2 Figure 1. X-chromosome wide analyses reveal two genome-wide significant loci in the

3 combined sex meta-analysis. X-chromosome wide sex-stratified discovery analyses in males (A)

4 and in females (B), and combined sex meta-analysis (C). The most significantly regulated gene in

5 brain tissues (RPL10) or the nearest gene (GPM6B, PJA1, PTCHD1, RTL9) is annotated on top of

6 the lead SNP at each locus. The red line is the genome-wide significance threshold $\left(\mathrm{p}<5 \times 10^{-8}\right)$

7 and the blue line is the suggestive significance threshold $\left(\mathrm{p}<10^{-5}\right)$, corresponding to $\mathrm{X}$ -

8 chromosome-wide correction. 

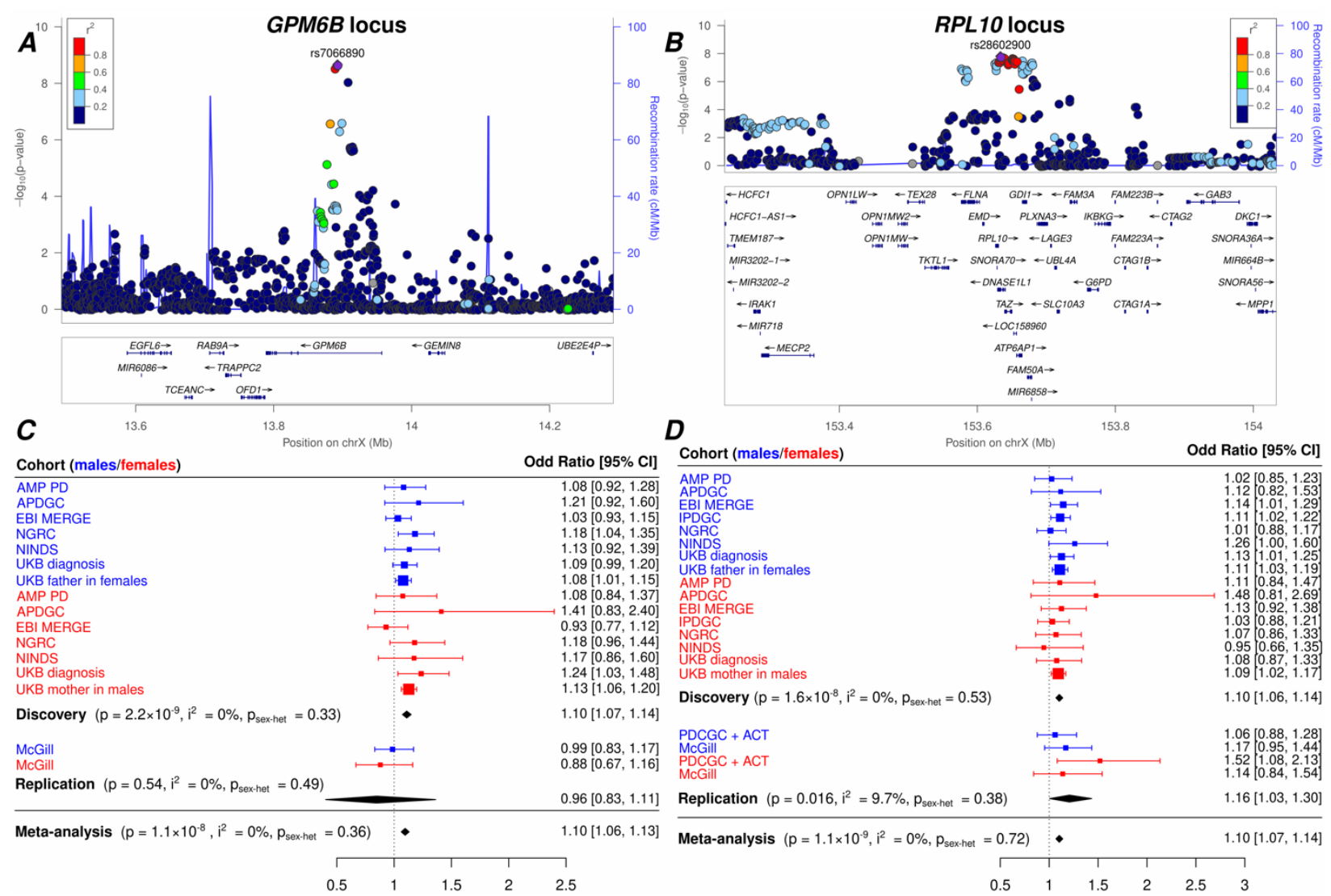

Figure 2. Regional details and forest plots of the genome-wide significant loci in the combined

3 sex meta-analysis. Locuszoom plots of the GPM6B (A) and RPL10 (B) loci, and the forest plots

4 for their respective lead variant emphasizing their consistent direction of effect across datasets $(\mathbf{C}$, 5 D). 

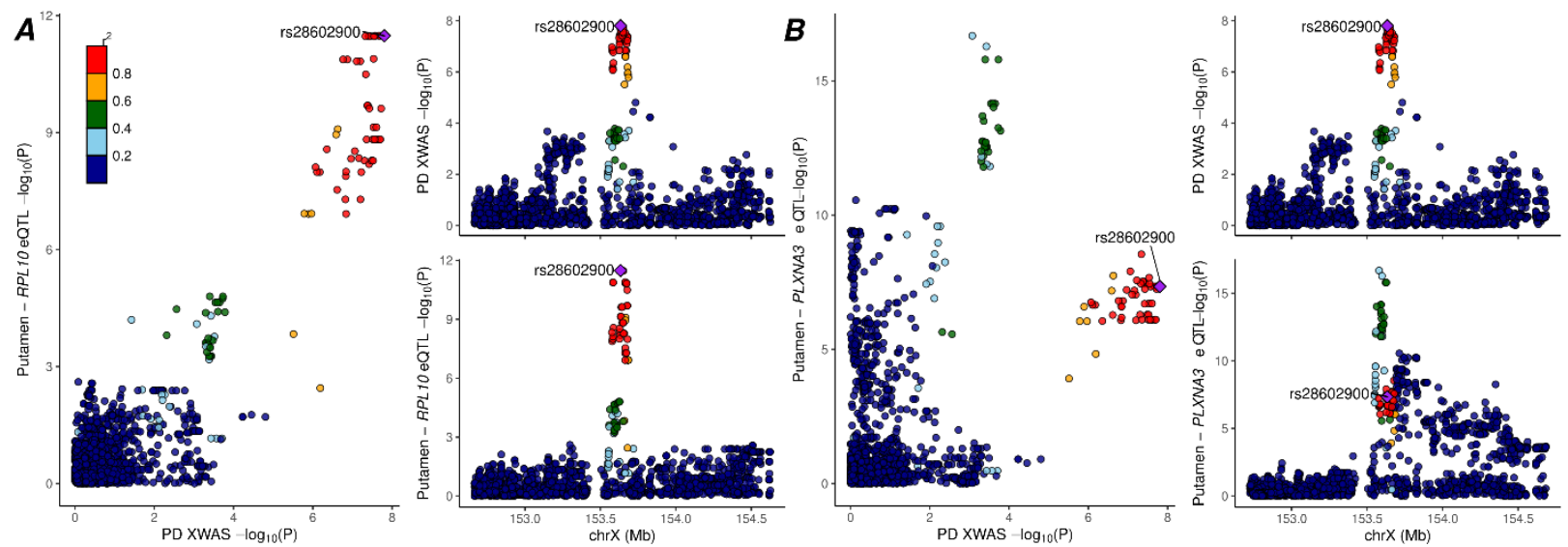

Figure 3. Colocalization analyses between association with PD risk and association with gene

expression in brain tissues. (A) The locus, on Xq28, associated with PD risk colocalized with the RPL10 eQTL in the putamen (and in the ten other GTEx brain tissues with FDR-significant

$5 \quad$ RPL10 eQTLs, data not shown). (B) The locus, on Xq28, associated with PD risk did not colocalize with the PLXNA3 eQTL in the putamen (or in other brain tissues with FDR-significant PLXNA3 eQTLs). Rather, the eQTL signal is driven by a nearby eQTL locus in low linkage disequilibrium

8 with the PD risk locus. 
medRxiv preprint doi: https://doi.org/10.1101/2020.12.18.20248459; this version posted December 20, 2020. The copyright holder for this preprint (which was not certified by peer review) is the author/funder, who has granted medRxiv a license to display the preprint in perpetuity.

It is made available under a CC-BY-NC 4.0 International license .

1 Table 1. Overview of the cohorts included in the Parkinson's disease discovery meta-analysis.

2 N SNPS pre-IMP: number of SNPs passing quality control prior to imputation, or number of SNPs

3 in the WGS. N SNPS post-IMP: number of SNPs with imputation quality $\mathrm{r}^{2}>0.3(\mathrm{~N})$ and after

4 filtering for MAF $>0.01 \&$ genotyping rate $>95 \%$. NWE: Northwestern Europeans.

\begin{tabular}{|c|c|c|c|c|c|c|c|}
\hline Cohort & $\begin{array}{l}\text { Genotyping } \\
\text { platform }\end{array}$ & $\begin{array}{l}\text { N SNPs } \\
\text { pre-IMP }\end{array}$ & $\begin{array}{l}\text { N SNPs } \\
\text { post-IMP }\end{array}$ & $\begin{array}{l}\mathrm{N}- \\
\text { NWE }\end{array}$ & $\begin{array}{l}\% \text { - } \\
\text { Cases }\end{array}$ & $\begin{array}{l}\mu(\sigma)- \\
\text { age cases }\end{array}$ & $\begin{array}{l}\mu(\sigma)- \\
\text { age controls }\end{array}$ \\
\hline IPDGC & $\begin{array}{l}\text { Illumina } \\
\text { NeuroX Array }\end{array}$ & 1,664 & 26,276 & & & & \\
\hline Females & & & & 3,103 & 45.0 & $66.3(14.1)$ & $60.7(13.0)$ \\
\hline Males & & & & 4,571 & 51.9 & $62.5(16.1)$ & $59.2(12.4)$ \\
\hline NINDS & $\begin{array}{l}\text { Illumina } \\
\text { HumanHap550 }\end{array}$ & 19,788 & 223,218 & & & & \\
\hline Females & & & & 717 & 45.0 & $62.3(14.6)$ & $66.43(11.0)$ \\
\hline Males & & & & 724 & 62.4 & $55.4(17.6)$ & $65.52(11.5)$ \\
\hline APDGC & $\begin{array}{l}\text { Illumina } \\
\text { HumanOmni1-Quad }\end{array}$ & 17,496 & 224,713 & & & & \\
\hline Females & & & & 263 & 53.0 & $78.1(13.8)$ & $77.2(8.4)$ \\
\hline Males & & & & 497 & 74.0 & $86.4(11.0)$ & $79.5(7.9)$ \\
\hline NGRC & $\begin{array}{l}\text { Illumina } \\
\text { HumanOmni1-Quad }\end{array}$ & 11,732 & 225,792 & & & & \\
\hline Females & & & & 1,704 & 33.7 & $70.9(13.9)$ & $67.17(10.6)$ \\
\hline Males & & & & 1,921 & 62.5 & $70.2(14.3)$ & $67.11(11.3)$ \\
\hline EBI MERGE & $\begin{array}{l}\text { Illumina } 610 \mathrm{k} \text { Quad } \\
\text { \& Illumina } 1.2 \mathrm{M}\end{array}$ & 5,727 & 217,464 & & & & \\
\hline Females & & & & 2,038 & 32.6 & NA & NA \\
\hline Males & & & & 2,561 & 43.2 & NA & NA \\
\hline AMP-PD WGS & WGS & $2,466,931$ & 221,601 & & & & \\
\hline Females & & & & 1,005 & 58.4 & $64.0(9.3)$ & $62.0(11.1)$ \\
\hline Males & & & & 1,412 & 73.4 & $64.7(9.5)$ & $63.9(11.2)$ \\
\hline $\begin{array}{l}\text { UK Biobank } \\
\text { PD diagnosis }\end{array}$ & $\begin{array}{l}\text { Affymetrix } \\
\text { Axiom Array }\end{array}$ & $\begin{array}{l}\text { Cf Bycroft } \\
\text { et al. (2018) }\end{array}$ & 261,450 & & & & \\
\hline Females & & & & 147,263 & 0.27 & NA & NA \\
\hline Males & & & & 128,981 & 0.55 & NA & NA \\
\hline $\begin{array}{l}\text { UK Biobank } \\
\text { Proxy-PD }\end{array}$ & $\begin{array}{l}\text { Affymetrix } \\
\text { Axiom Array }\end{array}$ & $\begin{array}{l}\text { Cf Bycroft } \\
\text { et al. }(2018)\end{array}$ & 261,450 & & & & \\
\hline Mother PD in males & & & & 128,275 & 1.4 & NA & NA \\
\hline Father PD in females & & & & 146,867 & 2.4 & NA & NA \\
\hline
\end{tabular}


medRxiv preprint doi: https://doi.org/10.1101/2020.12.18.20248459; this version posted December 20, 2020. The copyright holder for this preprint (which was not certified by peer review) is the author/funder, who has granted medRxiv a license to display the preprint in perpetuity.

It is made available under a CC-BY-NC 4.0 International license .

1 Table 2. Overview of the cohorts included in the Parkinson's disease replication meta-

2 analysis. $N$ SNPS pre-IMP: number of SNPs passing quality control prior to imputation, or number

3 of SNPs in the WGS. N SNPs post-IMP: number of SNPs with imputation quality $\mathrm{r}^{2}>0.3(\mathrm{~N})$ and

4 after filtering for MAF $>0.01 \&$ genotyping rate $>95 \%$. NWE: Northwestern Europeans.

\begin{tabular}{|c|c|c|c|c|c|}
\hline Cohort & $\begin{array}{l}\text { Genotyping } \\
\text { platform }\end{array}$ & $\begin{array}{l}\text { N SNPs } \\
\text { pre-IMP }\end{array}$ & $\begin{array}{l}\text { N SNPs } \\
\text { post-IMP }\end{array}$ & $\begin{array}{l}\text { N - } \\
\text { NWE }\end{array}$ & $\begin{array}{l}\%- \\
\text { Cases }\end{array}$ \\
\hline PDCGC \& ACT & $\begin{array}{l}\text { Illumina NeuroX } \\
\& \text { Illumina } 660 \mathrm{~W}\end{array}$ & $\begin{array}{l}1,322 \text { in PDCGC } \\
8,908 \text { in ACT }\end{array}$ & 29,283 in both & & \\
\hline Females & & & & 1,099 & 20.2 \\
\hline Males & & & & 1,141 & 36.5 \\
\hline McGill & $\begin{array}{l}\text { Illumina } \\
\text { Infinium OmniExpress-24 }\end{array}$ & 15,912 & 227,144 & & \\
\hline Females & & & & 758 & 41.7 \\
\hline Males & & & & 1,033 & 59.1 \\
\hline
\end{tabular}


Table 3. Lead SNPs in loci passing the suggestive threshold $\left(\mathrm{P}<10^{-5}\right)$ in the combined sex meta-analysis or single-sex metaanalyses. $\mathrm{m} / \mathrm{M}$ : minor allele/major allele; MAF: minor allele frequency; P- het: P-value from the sex-heterogeneity test, ORs are calculated with respect to the minor allele. SNPs on lines highlighted in bold are genome-wide significant $\left(\mathrm{P}<5 \times 10^{-8}\right)$. disc: discovery meta-analysis; repl: replication meta-analysis; meta: full meta-analysis.

\begin{tabular}{|c|c|c|c|c|c|c|c|c|c|c|c|c|c|c|c|}
\hline SNP & $\begin{array}{c}\text { Pos } \\
\text { (chrX/hg19) }\end{array}$ & $\begin{array}{l}\text { Alleles } \\
(\mathbf{m} / \mathbf{M})\end{array}$ & MAF & $\begin{array}{c}\text { Nearest } \\
\text { Gene }\end{array}$ & Sex & $\begin{array}{l}\text { OR } \\
\text { disc }\end{array}$ & $\begin{array}{c}95 \% \text { CI } \\
\text { disc }\end{array}$ & $\begin{array}{c}\mathrm{P} \\
\text { disc }\end{array}$ & $\begin{array}{l}\text { OR } \\
\text { repl }\end{array}$ & $\begin{array}{c}95 \% \text { CI } \\
r e p l\end{array}$ & $\begin{array}{c}\mathrm{P} \\
\text { repl }\end{array}$ & $\begin{array}{c}\text { OR } \\
\text { meta }\end{array}$ & $\begin{array}{c}95 \% \text { CI } \\
\text { meta }\end{array}$ & $\begin{array}{c}\mathrm{P} \\
\text { meta }\end{array}$ & P-het \\
\hline \multirow[t]{3}{*}{ rs7066890 } & 13892582 & $\mathrm{~T} / \mathrm{C}$ & 0.155 & GРМ6В & Combined sex & 1.10 & $1.07-1.14$ & $2.2 \times 10^{-9}$ & 0.96 & 0.83-1.11 & 0.54 & 1.10 & 1.06-1.13 & $1.1 \times 10^{-8}$ & 0.36 \\
\hline & $\mathrm{Xp} 22.2$ & & & & Females & 1.12 & $1.07-1.18$ & $4.7 \times 10^{-6}$ & 0.88 & $0.67-1.16$ & 0.37 & 1.12 & $1.06-1.17$ & $1.4 \times 10^{-5}$ & \\
\hline & & & & & Males & 1.09 & $1.04-1.14$ & $7.0 \times 10^{-5}$ & 0.99 & $0.83-1.17$ & 0.87 & 1.08 & 1.04-1.14 & $1.3 \times 10^{-4}$ & \\
\hline \multirow[t]{3}{*}{ rs111841435 } & 23342372 & $\mathrm{C} / \mathrm{T}$ & 0.144 & PTCHD1 & Combined sex & 1.07 & $1.03-1.11$ & $9.4 \times 10^{-5}$ & 0.95 & $0.81-1.11$ & 0.49 & 1.06 & $1.03-1.10$ & $2.3 \times 10^{-4}$ & 0.005 \\
\hline & Xp22.11 & & & & Females & 1.00 & $0.95-1.06$ & 0.89 & 0.88 & $0.64-1.21$ & 0.43 & 1.00 & $0.95-1.06$ & 1.00 & \\
\hline & & & & & Males & 1.11 & $1.07-1.16$ & $1.3 \times 10^{-6}$ & 0.97 & $0.81-1.16$ & 0.87 & 1.10 & $1.06-1.15$ & $3.6 \times 10^{-6}$ & \\
\hline \multirow[t]{3}{*}{ rs11796528 } & 68378849 & $\mathrm{~A} / \mathrm{G}$ & 0.169 & PJA1 & Combined sex & 1.08 & $1.05-1.12$ & $3.2 \times 10^{-6}$ & 1.04 & $0.89-1.21$ & 0.61 & 1.08 & $1.05-1.11$ & $1.1 \times 10^{-6}$ & 0.81 \\
\hline & Xq13.1 & & & & Females & 1.08 & $1.02-1.13$ & $6.6 \times 10^{-3}$ & 1.00 & $0.75-1.35$ & 0.98 & 1.07 & $1.02-1.13$ & $4.3 \times 10^{-3}$ & \\
\hline & & & & & Males & 1.09 & $1.04-1.13$ & $1.5 \times 10^{-4}$ & 1.05 & $0.89-1.25$ & 0.56 & 1.08 & $1.04-1.13$ & $7.5 \times 10^{-5}$ & \\
\hline \multirow[t]{3}{*}{ rs41306249 } & 10969443 & $\mathrm{~T} / \mathrm{C}$ & 0.047 & RTL9 & Combined sex & 1.13 & $1.07-1.19$ & $6.2 \times 10^{-6}$ & 1.81 & $1.40-2.36$ & $5.7 \times 10^{-6}$ & 1.15 & $1.09-1.21$ & $9.0 \times 10^{-8}$ & 0.87 \\
\hline & Xq23 & & & & Females & 1.13 & $1.04-1.24$ & $5.5 \times 10^{-3}$ & 2.12 & $1.38-3.27$ & $6.3 \times 10^{-3}$ & 1.16 & $1.06-1.26$ & $6.8 \times 10^{-4}$ & \\
\hline & & & & & Males & 1.13 & $1.06-1.21$ & $3.6 \times 10^{-4}$ & 1.67 & $1.21-2.30$ & $1.8 \times 10^{-3}$ & 1.15 & $1.08-1.23$ & $3.6 \times 10^{-5}$ & \\
\hline \multirow[t]{3}{*}{ rs28602900 } & 153633533 & $\mathbf{A} / \mathbf{G}$ & 0.115 & RPL10 & Combined sex & 1.10 & $1.06-1.14$ & $1.6 \times 10^{-8}$ & 1.16 & $1.03-1.30$ & 0.016 & 1.10 & $1.07-1.14$ & $1.1 \times 10^{-9}$ & 0.72 \\
\hline & Xq28 & & & & Females & 1.09 & $1.03-1.14$ & $3.0 \times 10^{-3}$ & 1.29 & $1.03-1.62$ & 0.026 & 1.09 & $1.04-1.15$ & $6.7 \times 10^{-4}$ & \\
\hline & & & & & Males & 1.11 & $1.06-1.16$ & $1.2 \times 10^{-6}$ & 1.11 & $0.97-1.27$ & 0.14 & 1.11 & $1.07-1.15$ & $4.0 \times 10^{-7}$ & \\
\hline
\end{tabular}

